\title{
High Efficacy of Rapid Urine Test for Diagnosis of Helicobacter pylori Infection in Thai People
}

\author{
Natsuda Aumpan ${ }^{1}$, Ratha-Korn Vilaichone ${ }^{1,2,3 *}$, Peranart Chotivitayatarakorn ${ }^{1,2,3}$, \\ Bubpha Pornthisarn ${ }^{1}$, Soonthorn Cholprasertsuk ${ }^{1}$, Patommatat Bhanthumkomol ${ }^{1}$, \\ Amornnivit Kanokwanvimol ${ }^{1}$, Sith Siramolpiwat ${ }^{1,3}$, Varocha Mahachai $^{2,3,4}$
}

\begin{abstract}
Background: Accurate diagnosis of Helicobacter pylori $(H$. pylori) infection plays an important role in further effective treatment. Rapid urine test (RAPIRUN) is a test developed for qualitative detection of urine H. Pylori antibody and use for determine the sensitivity, specificity and accuracy. However, the test needs validation in Thai population before using in clinical practice. Objective: This study aimed to compare performance of different diagnostic tests on H. pylori detection in Thai population. Methods: Total of 94 patients with dyspepsia who referred to Thammasat University Hospital, Pathumthani, Thailand, between December 2012 and April 2013 were enrolled in this study. All patients underwent gastroscopy. Then, 3 biopsies at antrum were taken for H. pylori diagnosis. including rapid urease test (Pronto Dry, Eisai, Thailand), H. pylori culture, and histopathology. Urine samples were also collected at the same time for rapid urine test (RAPIRUN H. pylori Antibody, Otsuka Pharmaceutical Co., Ltd.). Patients were diagnosed with H. pylori-positive if their culture or rapid urease tests plus histopathology yielded positive results. Results: Total of 29 patients $(30.9 \%)$ were infected with H. pylori. Prevalence of $H$. pylori infection by rapid urease test, histopathology, culture and rapid urine test were $25.5 \%, 28.7 \%, 29.8 \%$, and $32.9 \%$ respectively. We observed that rapid urease test, histopathology, culture, and rapid urine test had sensitivity of $82.8 \%, 93.1 \%, 93.1 \%$ and $86.2 \%$; specificity of $100 \%$, $100 \%, 100 \%$, and $90.8 \%$; and accuracy of $95.7 \%, 97.9 \%, 97.9 \%$, and $89.4 \%$, respectively. Conclusion: Rapid urine test (RAPIRUN) provided a reliable result for diagnosis of $H$. pylori infection. Furthermore, this rapid urine test demonstrated high accuracy, reliable, safe handle and easy to use. We suggested rapid urine test for diagnosis of $H$. pylori infection in Thai population since we found it less invasive and with higher reliable efficacy.
\end{abstract}

Keywords: Rapid urine test- Helicobacter pylori- Thailand

Asian Pac J Cancer Prev, 20 (5), 1525-1529

\section{Introduction}

Helicobacter pylori (H. pylori), a spiral-shaped, gram-negative bacterium, is generally found in deep mucous layer of gastric epithelium. Both bacterial and host factors influence the $H$. pylori pathogenicity. Bacterial attachment to gastric cell surface and subsequent release of enzymes can cause direct cellular damage. In addition, the bacteria also trigger the host inflammatory response which leads to further tissue injury (Kusters et al., 2006). Gastric epithelial destruction by $H$. pylori infection resulted in many conditions such as peptic ulcer diseases, chronic gastritis, gastric adenocarcinoma, and gastric mucosa-associated lymphoid tissue (MALT) lymphoma (McColl, 2010; Srinarong et al., 2014; Chotivitayatarakorn et al., 2017; Vilaichone et al., 2017). Until now, a large number of research has proven that chronic inflammation caused by $H$. pylori is associated with gastric cancer (Parsonnet et al., 1991; Huang et al., 1998; Vilaichone et al., 2014). Furthermore, recent studies have emphasized the importance of $H$. pylori eradication which can decrease gastric cancer incidence (Hamajima et al., 2004; Lee et al., 2016). Overall, H. pylori testing and eradication are recommended in high-risk patients such as those with family history of gastric cancer in first degree relative, peptic ulcer diseases, and dyspepsia not responding to treatment (Mahachai et al., 2016).

Accurate diagnosis of $H$. pylori infection plays a pivotal role in further effective treatment. Several diagnostic tests for $H$. pylori have been developed since its first isolation in 1983. Tests are classified as invasive or non-invasive. Invasive tests which are endoscopy-based

${ }^{1}$ Gastroenterology Unit, Department of Medicine, Faculty of Medicine, Thammasat University Hospital, ${ }^{2}$ National Digestive Diseases Research Center of Thailand, ${ }^{3}$ Department of Medicine, Chulabhorn International College of Medicine (CICM) at Thammasat University, Pathumthani, ${ }^{4}$ Gastrointestinal and Liver Center, Bangkok Medical Center, Bangkok, Thailand. *For Correspondence: Vilaichone@hotmail.co.th 
include histology, rapid urease test, culture, and polymerase chain reaction (PCR); whereas, non-invasive tests include serology, urea breath test, and stool antigen test (Patel et al., 2014). Nowadays, researchers tend to develop more sensitive but less invasive diagnostic tests. Rapid urine test (RAPIRUN) was invented by Otsuka Pharmaceutical Co., Ltd. (Tokyo, Japan) using immunochromatographic assay for qualitative detection of urine $H$. pylori antibody. Having been approved by FDA in 2006, RAPIRUN was evaluated in many countries and high sensitivity, specificity, and accuracy was reported (Graham and Reddy, 2001; Syam et al., 2015). However, the test needs validation in Thai population before its usage in clinical practice.

This study aimed to compare performance of different diagnostic tests for H. pylori detection in Thai population. The investigated diagnostic test were rapid urease test, histopathology, and culture and rapid urine test.

\section{Materials and Methods}

\section{Patients}

Total of 94 patients with dyspepsia (50 males and 44 females, with the mean age of 43.2 years) who referred to Thammasat University Hospital, Pathumthani, Thailand, between December 2012 and April 2013 were enrolled in this study. Inclusion criteria were aging more than 18 years and lack of receiving $H$. pylori treatment. Patients who received $\mathrm{H} 2$ receptor antagonists, proton pump inhibitor (PPI), bismuth compound, and antimicrobial agents in the past one month were excluded. Furthermore, we excluded patients receiving NSAIDs and anticoagulation drugs or had history of stomach surgery. Patients with significant co-morbid diseases such as renal failure and congestive heart failure, pregnant women, or breastfeeding women were not included in this study either. Informed consent was obtained from all patients before entering the study.

\section{H. pylori diagnosis}

Gastroscopy was carried out for all patients . During the endoscopy, 3 antral biopsies were obtained for rapid urease test (Pronto Dry, Eisai, Thailand), histopathology, and $H$. pylori culture. Urine samples were also collected at the same time for rapid urine test (RAPIRUN by Otsuka Pharmaceutical Co., Ltd., Tokyo, Japan). Positive gold standard tests were defined as (1) positive rapid urease test plus histopathology or (2) culture positive H. Pylori infection.

\section{The Rapid Urine Test}

After collecting urine samples, they were immediately sent to the laboratory within 4 hours of collection. Next, $0.5 \mathrm{cc}$ of each fresh urine was stirred with $0.5 \mathrm{cc}$ of diluent by pipette. The test stick (contained dried antihuman IgG antibody-binding gold colloidal particles and nitrocellulose membrane) was then dipped into the mixture of urine and diluent. H. pylori antigen and antihuman IgG antibodies were immobilized in the membrane to produce a test line and a control line, respectively. If patient had human IgG antibodies in urine sample, they will diffuse and form human IgG-antihuman IgG antibody complexes producing a red band by immunochromatographic technique. When two red bands were present at the test line and control line within 20 minutes, the test was considered positive. When only the control line was observed, the test was considered negative. If no line was observed, the test was regarded invalid caused by technical errors or extremely diluted urine.

\section{Statistical analysis}

All data were analysed by SPSS version 19 (SPSS Inc., Chicago, IL, USA). The demographic data were analysed by using the Fisher's exact test or chi-squared where appropriate. P-values less than 0.05 were considered as statistically significance.. The study protocol was in accordance with clinical practice guidelines and it was approved by our university ethics committee.

\section{Results}

A total of 94 patients with dyspeptic symptoms were enrolled in this study (50 males and 44 females with the mean age of 43.2 years old). Among 94 patients, 29 patients $(30.9 \%)$ were diagnosed with $H$. pylori infection by gold standard tests. Positive test was found in 24 patients $(25.5 \%)$ for rapid urease test (RUT), 27 patients

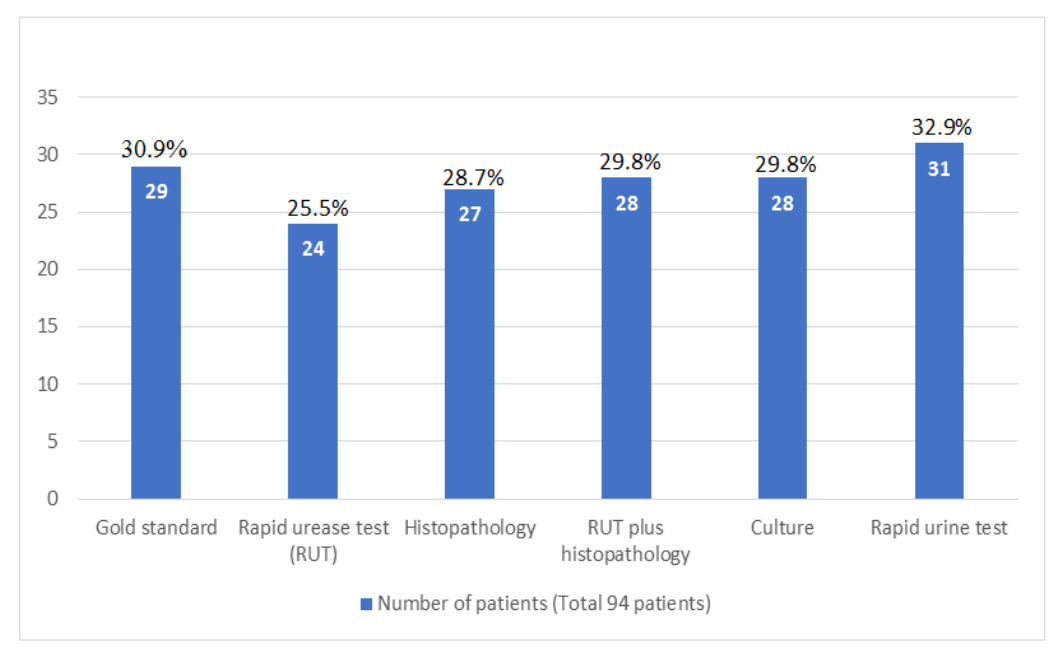

Figure 1. Prevalence of H. pylori Infection by Different Tests 
Table 1. Efficacy of Rapid Urine Test Compare to Gold Standard for H. pylori Diagnosis

\begin{tabular}{lccc}
\hline Test & $\begin{array}{c}\text { Gold standard } \\
\text { test }+\end{array}$ & $\begin{array}{c}\text { Gold standard } \\
\text { test - }\end{array}$ & Total \\
\hline Rapid urine test + & 25 & 6 & 31 \\
Rapid urine test - & 4 & 59 & 63 \\
Total & 29 & 65 & 94 \\
\hline
\end{tabular}

(28.7\%) for histopathology, 28 patients $(29.8 \%)$ for rapid urease test plus histopathology, 28 patients $(29.8 \%)$ for culture, and 31 patients $(32.9 \%)$ for rapid urine test. There was one patient who had negative culture but positive rapid urease test and histopathology and considered positive for gold standard tests. All data are demonstrated in the Figure 1.

Among 29 patients with positive gold standard tests, 25 had positive rapid urine test. There were 6 patients with positive rapid urine test, but they had negative gold standard tests (Table 1).

The rapid urine test had sensitivity of $86.2 \%$, specificity of $90.8 \%$, and accuracy of $89.4 \%$. whereas, rapid urease test, histopathology, and culture had sensitivity of $82.8 \%, 93.1 \%, 93.1 \%$; specificity of $100 \%$, $100 \%, 100 \%$; and accuracy of $95.7 \%, 97.9 \%, 97.9 \%$, respectively. In our study population, the rapid urine test had negative predictive value of $93.7 \%$ and positive predictive value of $80.6 \%$.

\section{Discussion}

So far, there has been much effort to develop ideal diagnostic tests for $H$. pylori detection. Several non-invasive diagnostic methods have been tested and compared with reference standard in search of the best non-invasive test for $H$. pylori infection. Current non-invasive tests consist of ${ }^{13} \mathrm{C}$ or $1{ }^{4} \mathrm{C}$ urea breath test, stool antigen test, and antibody tests in serum, urine, and saliva (Patel et al., 2014; Tongtawee et al., 2016). In 2018, using Cochrane database of systematic reviews, researchers included 101 studies involving 11,003 participants to compare 3 non-invasive tests that were urea breath test, stool antigen test, and serology against the gold standard, but they excluded urine and saliva-based tests because they were not commonly used. They revealed that urea breath tests had the highest sensitivity for $13 \mathrm{C}$ or $14 \mathrm{C}$ urea breath test (94\% and $92 \%$, respectively), followed by $84 \%$ for serology and $83 \%$ for stool antigen test. They also concluded that urea breath test had higher diagnostic accuracy than serology and stool antigen test (Best et al., 2018). Although many studies have considered urea breath test as the mostly used and accurate non-invasive test, these tests have some limitation such as high cost and poor sensitivity in patients with gastric surgery history or those receiving PPI, H2 receptor antagonists, bismuth, and antibiotics (Gatta et al., 2004; Wang et al., 2015). The monoclonal stool antigen test was another proven method that had high sensitivity and accuracy and could be used both for diagnosis or evaluation after $H$. pylori eradication (Gisbert et al., 2006). However, a stool test result can be unreliable if a patient has gastrointestinal bleeding (Hanvivatvong et al., 2006). Another non-invasive test is serology which is not affected by gastrointestinal bleeding or the use of PPI or antibiotic. Nevertheless, it has limitation in distinguishing between active or past infection because of persistence of $H$. pylori antibodies up to 6-12 months after successful treatment (Gisbert and Abraira, 2006). Owing to some disadvantages of the above-mentioned non-invasive tests, the urine test has been verified in many countries to find whether it is comparable to current standard methods.

In our study, we validated rapid urine test for the diagnosis of $H$. pylori infection in Thai people and as a non-invasive method and meriting with high sensitivity, specificity, and accuracy according to previous studies (Graham and Reddy, 2001; Quach et al., 2014; Syam et al., 2015). There are two types of urine tests for diagnosis of $H$. pylori infection. The first one is urine ELISA for anti- $H$. pylori $\mathrm{IgG}$ discovered in 1993 with sensitivity of $95.9 \%$ and specificity of $90 \%$ (Alemohammad et al., 1993). The other type of urine-based test is rapid urine test which uses immunochromatographic method for detection of anti- $H$. pylori IgG in urine (Graham and Reddy, 2001). One study showed that accuracy of urine ELISA (93.2\%) was slightly higher than that of the immunochromatographic method (91.2\%), and both were comparable to serological tests but superior to stool antigen test (83.5\%) (Adachi et al., 2002). Results from our study showed that rapid urine test could detect $H$. Pylori approximately with the same prevalence $(32.9 \%)$ as the gold standard tests $(30.9 \%)$. Moreover, we also found that the rapid urine test had comparable sensitivity, specificity, and accuracy $(86.2,90.8 \%$, and $89.4 \%$, respectively) to previous studies from other Southeast Asian countries such as Vietnam $(84.7 \%, 89.9 \%$, and $87.0 \%$, respectively) and Indonesia $(83.3 \%, 94.7 \%$, and 93.2\%, respectively) (Quach et al., 2014; Syam et al., 2015). However, our study revealed lower sensitivity, specificity, and accuracy of the rapid urine test than those reported in two studies from Japan $(93.7 \%, 88.9 \%$, and $92.2 \%$, respectively) and China $(96.7 \%, 95.2 \%$, and 95.9\%, respectively) (Fujisawa et al., 2001; Wong et al., 2002). This discrepancy maybe because of different H. pylori strains between Northeast and Southeast Asia. The vacA $\mathrm{m} 1$ type strains were predominant in Japan and South Korea while $\mathrm{m} 2$ type strains were more common in Southeast Asia such as Vietnam and Taiwan (Yamaoka et al., 2002). The urine test, which was developed from DNA analyses from Japaneses $H$. pylori genes, could produce different test results. Another advantage of using rapid urine test is easily obtainable and non-invasive specimen collection. Moreover, given that the urine test does not require centrifugation, it takes less time.

Results from our study is considered applicable to Thai dyspeptic patients. Although there were few limitations in this study. In order to develop rapid urine test, Japanese H. Pylori strain OHPC-040 was used because it revealed the strongest reaction for positive specimen (Katsuragi et al., 1998). As a result, different H. Pylori strains in Thai patients and divergent genetic background could induce varying antigen-antibody response and possibly led to 
false negative results in our study (Uchida et al., 2015; Vilaichone et al., 2018). Six patients with false positive result could also be explained by extended positive urine test after H. pylori eradication (Graham and Reddy, 2001). Moreover, the sample size in this study was relatively small. Larger studies may be needed to ascertain the validity of the rapid urine test in the future.

In conclusion, Rapid urine test (RAPIRUN) provides a reliable result for diagnosis of $H$. pylori infection. Furthermore, this rapid urine test, according to our findings, has high accuracy and it was reliable, safe handle, and easy to use. The rapid urine test is not only comparable to gold standard tests but also non-invasive tests and it is suggested for Thai people.

\section{Acknowledgements}

This study was supported by the National Gastric Cancer and Gastrointestinal Diseases Research Center, Pathumthani, Thailand.

\section{References}

Adachi K, Kawamura A, Ono M, et al (2002). Comparative evaluation of urine-based and other minimally invasive methods for the diagnosis of Helicobacter pylori infection. J Gastroenterol, 37, 703-708.

Alemohammad MM, Foley TJ, Cohen H (1993). Detection of immunoglobulin $\mathrm{G}$ antibodies to Helicobacter pylori in urine by an enzyme immunoassay method. J Clin Microbiol, 31, 2174-7.

Best LM, Takwoingi Y, Siddique S, et al (2018). Non-invasive diagnostic tests for Helicobacter pylori infection. Cochrane Database Syst Rev, doi: 10.1002/14651858.CD012080. pub2.

Chotivitayatarakorn P, Mahachai V, Vilaichone RK (2017). Effectiveness of 7-day and 14-day MoxifloxacinDexlansoprazole based triple therapy and probiotic supplement for Helicobacter Pylori eradication in Thai patients with non-ulcer dyspepsia: A double-blind randomized placebo-controlled study Asian Pac J Cancer Prev, 18, 2839-43.

Fujisawa T, Kaneko T, Kumagai T, et al (2001). Evaluation of urinary rapid test for Helicobacter pylori in general practice. J Clin Lab Anal, 15, 154-9.

Hanvivatvong O, Thong-Ngam D, Kuakarn S, et al (2006). Evaluation of Helicobacter pylori stool antigen test in Thai patients with upper gastrointestinal bleeding. J Med Assoc Thai, 89, 98-103.

Gatta L, Vakil N, Ricci C, et al (2004). Effect of proton pump inhibitors and antacid therapy on $13 \mathrm{C}$ urea breath tests and stool test for Helicobacter pylori infection. Am J Gastroenterol, 99, 823-9.

Gisbert JP, de la Morena F, Abtaira V (2006). Accuracy of monoclonal stool antigen test for the diagnosis of H. pylori infection: a systematic review and meta-analysis. Am J Gastroenterol, 101, 1921-30.

Gisbert JP, Abraira V (2006). Accuracy of Helicobacter pylori diagnostic tests in patients with bleeding peptic ulcer: a systematic review and meta-analysis. Am J Gastroenterol, 101, 848-63.

Graham DY, Reddy S (2001). Rapid detection of anti-Helicobacter pylori IgG in urine using immunochromatography. Aliment Pharmacol Ther, 15, 699-702.

Hamajima N, Goto Y, Nishio K, et al (2004). Helicobacter pylori eradication as a preventive tool against gastric cancer. Asian Pac J Cancer Prev, 5, 246-252.

Huang JQ, Sridhar S, Chen Y, et al (1998). Meta-analysis of the relationship between Helicobacter pylori seropositivity and gastric cancer. Gastroenterology, 114, 1169-1179.

Katsuragi K, Noda A, Tachikawa T, et al (1998). Highly sensitive urine-based enzyme-linked immunosorbent assay for detection of antibody to Helicobacter pylori. Helicobacter, 3, 289-295.

Kuster JG, van Vilet AHM, Kuipers EJ (2006). Pathogenesis of Helicobacter pylori Infection. Clin Microbiol Rev, 19, 449-90.

Lee YC, Chiang TH, Chou CK, et al (2016). Association between Helicobacter pylori eradication and gastric cancer incidence: A systematic review and meta-analysis. Gastroenterology, 150, 1113-24.

Mahachai V, Vilaichone RK, Pittayanon R, et al (2016). Thailand consensus on Helicobacter pylori treatment 2015. Asian Pac $J$ Cancer Prev, 17, 2351-60.

McColl KE (2010). Helicobacter pylori Infection. $N$ Engl $J$ Med, 362, 1597-1604.

Parsonnet J, Friedman GD, Vandersteen DP, et al (1991). Helicobacter pylori infection and the risk of gastric carcinoma. N Engl J Med, 325, 1127-31.

Patel SK, Pratap CB, Jain AK, et al (2014). Diagnosis of Helicobacter pylori: What should be the gold standard?. World J Gastroenterol, 20, 12847-12859.

Quach DT, Hiyama T, Shimamoto F, et al (2014). Value of a new stick-type rapid urine test for the diagnosis of Helicobacter pylori infection in the Vietnamese population. World $J$ Gastroenterol, 20, 5087-91.

Srinarong C, Siramolpiwat S, Wongcha-um A, et al (2014). Improved eradication rate of standard triple therapy by adding bismuth and probiotic supplement for Helicobacter pylori treatment in Thailand. Asian Pac J Cancer Prev, 15, 9909-13.

Syam AF, Miftahussurur M, Uwan WB, et al (2015). Validation of urine test for detection of Helicobacter pylori infection in Indonesian population. Biomed Res Int, doi: 10.1155/2015/152823. Epub 2015 Dec 28.

Tongtawee T, Kaewpitoon S, Kaewpitoon N, et al (2016). Diagnosis of Helicobacter pylori infection. Asian Pac J Cancer Prev, 17, 1631-1635.

Vilaichone RK, Panarat W, Aekpongpaisit S, et al (2014). Clinical characteristics and Helicobacter pylori status of gastric cancer in Thailand. Asian Pac J Cancer Prev, 15, 9005-8.

Vilaichone RK, Quach DT, Yamaoka Y, et al (2018). Prevalence and pattern of antibiotic resistant strains of Helicobacter pylori infection in ASEAN. Asian Pac J Cancer Prev, 19, 1411-3.

Vilaichone RK, Wongcha Um A, Chotivitayatarakorn P (2017). Low re-infection rate of Helicobacter pylori after successful eradication in Thailand: A 2 years study. Asian Pac J Cancer Prev, 18, 695-7.

Wang YK, KUo FC, Liu CJ, etal (2015). Diagnosis of Helicobacter pylori infection: Current options and developments. World J Gastroenterol, 21, 11221-35.

Wong WM, Wong BC, Xia HH, et al (2002). An evaluation of a rapid urine test for the diagnosis of Helicobacter pylori infection in the Chinese population. Aliment Pharmaco Ther, 16, 813-7.

Uchida T, Miftahussurur M, Pittayanon R, et al (2015). Helicobacter pylori infection in Thailand: A nationwide study of the CagA Phenotype. PLoS One, 10. doi: 10.1371/ journal.pone.0136775. eCollection 2015.

Yamaoka Y, Orito E, Mizokami M, et al (2002). Helicobacter 
DOI:10.31557/APJCP.2019.20.5.1525

Rapid Urine Test for Diagnosis of Helicobacter Pylori Infection in Thailand

pylori in north and south America before columbus. FEBS

Lett, 517, 180-184.

\section{(ब) $(1)(8$}

This work is licensed under a Creative Commons Attribution-

Non Commercial 4.0 International License. 